\title{
Retailer Television Advertising: A Content Analysis of the Informational Cues Used By Retailers-An Abstract
}

\author{
Janna M. Parker and Bruce L. Alford
}

\begin{abstract}
In 2011, retailers spent $\$ 6.7$ billion on television advertising and over the last ten decades have maintained the number one or two spot for television advertising spending. Yet, the academic literature has focused on brands/product categories when conducting television advertising research and retailers have received little attention. The current study is an extension of Resnik and Sterns (1977) content analysis of TV advertising of brands to retailers. Their work has been replicated and extended but not with a sample which investigated retailers. Ailawadi et al. (2009) identified major differences in the goals, tools, and outcome measures used by retailers and manufacturers (brands). Thus, advertising strategies used by manufacturers should not be generalized to retailers and this research is needed to fill the gap. For this research, the sample contained ads from all goods-based retailers on the Leading 100 Advertisers' List from 2011. This study analyzed the framing of the advertisement and the advertisement was the unit of measurement. Videos of commercials found on YouTube were viewed for the content analysis. All ads downloaded from YouTube were the same version, no extended versions, as the ad shown on TV. YouTube was chosen as the source of the ads since many companies are now uploading their TV commercials immediately to YouTube. Whereas Resnik and Stern (1977) found just less than half (49.2\%) of ads to contain at least one informational cue, in the current sample of 179 ads, only one ad would be considered non-informative since it was the only one without any informational cues. Additional analysis included determining which criteria were used and if specific retailer types were likely to use certain criteria more than others.
\end{abstract}

References Available Upon Request.

\author{
J.M. Parker ( $\square)$ \\ James Madison University, Harrisonburg, VA, USA \\ e-mail: Parke4jm@jmu.edu \\ B.L. Alford \\ Louisiana Tech University, Ruston, LA, USA \\ e-mail: balford@latech.edu
}

